\title{
Relativistic equations of motion of massive bodies
}

\author{
Luc Blanchet \\ Institut D'Astrophysique de Paris \\ email: blanchet@iap.fr
}

\begin{abstract}
Highly relativistic equations of motions will play a crucial role for the detection and analysis of gravitational waves emitted by inspiralling compact binaries in detectors LIGO/ VIRGO on ground and LISA in space. Indeed these very relativistic systems (with orbital velocities of the order of half the speed of light in the last orbital rotations) require the application of a high-order post-Newtonian formalism in general relativity for accurate description of their motion and gravitational radiation [1]. In this contribution the current state of the art which has reached the third post-Newtonian approximation for the equations of motion [2-6] and gravitational waveform [7-9] has been described (see [10] for an exhaustive review). We have also emphasized the successful matching of the post-Newtonian templates to numerically generated predictions for the merger and ring-down in the case of black-hole binaries [11].
\end{abstract}

\section{References}

[1] Cutler, C., Apostolatos, T., Bildsten, L. Finn, L., Flanagan, E., Kennefick, D. Markovic, D. Ori, A. Poisson, E. Sussman, G., \& Thorne, K. 1993, The last three minutes: Issues in gravitational-wave measurements of coalescing compact binaries, Phys.Rev.Lett., 70, 2984

[2] Jaranowski, P. \& Schaefer, G. 1998, Third post-Newtonian higher order ADM Hamilton dynamics for two-body point-mass systems, Phys.Rev.D, 57, 7274

[3] Damour, T., Jaranowski, P., \& Schaefer, G. 2001, Dimensional regularization of the gravitational interaction of point masses, Phys.Lett.B, 513, 147

[4] Blanchet, L. \& Faye, G. 2001, General relativistic dynamics of compact binaries at the third post-Newtonian order, Phys.Rev.D, 63, 062005

[5] Andrade, V., de. Blanchet, L., \& Faye, G. 2001, Third post-Newtonian dynamics of compact binaries: Noetherian conserved quantities and equivalence between the harmonic-coordinate and ADM-Hamiltonian formalisms, Class.Quant.Grav., 18, 753

[6] Itoh, Y. \& Futamase, T. 2003, New derivation of a third post-Newtonian equation of motion for relativistic compact binaries without ambiguity, Phys.Rev.D, 68, 121501R

[7] Blanchet, L., Faye, G., Iyer, B., \& Joguet, B. 2002, Gravitational-wave inspiral of compact binary systems to $7 / 2$ post-Newtonian order, Phys.Rev.D, 65, 061501R

[8] Blanchet, L., Damour, T., Esposito-Farese, G., \& Iyer, B. 2004, Gravitational radiation from inspiralling compact binaries completed at the third post-Newtonian order, Phys.Rev.Lett., 93, 091101

[9] Blanchet, L., Faye, G., Iyer, B., \& Sinha, S. 2008, The third post-Newtonian gravitational wave polarisations and associated spherical harmonic modes for inspiralling compact binaries in quasi-circular orbits, Class.Quant.Grav., 25, 165003

[10] Blanchet, L. 2006, Gravitational radiation from post-Newtonian sources and inspiralling compact binaries, Liv.Rev. in Relat., 9, 4

[11] Buonanno, A., Cook, G., \& Pretorius, F. 2007, Inspiral, merger and ring-down of equal-mass black-hole binaries, Phys.Rev.D, 75, 124018 